\title{
1 Shortcut Method for Initialization of Dividing Wall Columns 2 and Estimating Pareto-Optimal NQ-curves
}

3 M.Sc. Lena-Marie Ränger ${ }^{1}$, Prof. Dr.-Ing. Thomas Grützner*1

$4{ }^{1}$ Laboratory of Thermal Process Engineering, Institute of Chemical Engineering, Ulm University, Albert-

$5 \quad$ Einstein-Allee 47, 89081 Ulm, Germany

$6 \quad{ }^{*}$ Corresponding author, thomas.gruetzner@uni-ulm.de

\section{Abstract}

In early project stages often no simulation results are available for dividing wall columns. Hence, shortcut methods are used to estimate suitable vapor and liquid splits. In a previous paper it was shown that $\dot{V}_{\min }$ diagrams are a suited tool to satisfy this need. However, it has turned out that it shows weaknesses for

11 columns with finite or non-optimal stages. This contribution closes that gap and presents an extended

12 approach to derive suited initial guesses. For this purpose, the original $\dot{V}_{\min }$ diagram is combined with a

13 heuristic approach to calculate Pareto-optimal column designs resulting in a stage-adapted $\dot{V}_{\min }$ diagram.

14 A comparative study shows that the new approach is a powerful tool to generate reliable guesses for 15 multiple dividing wall column simulations with finite stage numbers.

\section{Key Words}

17 - Dividing wall columns

18 - Shortcut

19 - Vmin diagram

20 - Vapor/liquid split

21 - Pareto-optimal 


\section{Introduction}

24 Distillation is one of the most important separation techniques in chemical industry [1-3]. This is contrasted by the fact that it is also one of the most energy-intensive process options. Accordingly, the process still offers a lot of scope for increasing efficiency. In this context dividing wall columns as intensified processes are a noteworthy alternative [4,5]. They can separate three, four or higher component mixtures into pure product flows in only one column shell by applying dividing walls to separate the feed and product sections [6]. Depending on the design variant of the column between 30 and $50 \%$ energy savings compared to conventional column sequences can be expected [7-9]. However, the modelling and operation of dividing wall columns is more complex compared to standard column sequences. In this context especially the vapor and liquid split above and below the dividing wall are relevant. Good initial estimates of these splits are important in three fields: First, for the operation of dividing wall columns suited ranges of the splits should be known in order to enable a stable column operation. Second, for the initialization of flowsheet simulation guesses for the internal streams are required. For bad initial guesses convergence problems are likely. Third, for the optimization of dividing wall columns initial simulations with solutions close to the demanded optimum are advantageous to find global optima. For all three cases, it is helpful to have a tool being able to estimate the split ranges reliably. In literature, the $\dot{V}_{\min }$ diagram is used for this task $[10,11]$. However, the diagram and thus also the predicted liquid and vapor splits are only valid at an infinite stage number since this is a basic assumption for its calculation. In a previous publication the authors of this contribution showed, that the suited vapor and liquid split ranges are strongly depending on the total stage number and allocation in the column sections [12]. Correspondingly, the suitability of the original $\dot{V}_{\min }$ method as an estimation tool decreases with decreasing stage number of the column. Accordingly, the objective of this paper is to present a new reliable method for the estimation of liquid and vapor splits for columns with finite stage numbers. This approach is also helpful in the case that the allocation of the theoretical stages inside the column is non-optimal for the separated mixture, which can occur for multi-purpose columns. The method is based on the extended version of the $\dot{V}_{\min }$ diagram, which was introduced in a previous publication [12]. In order to enable a shortcut calculation of the diagram, an approach to estimate Pareto-optimal compromises between stage number $N$ and energy requirement $\dot{Q}$ of distillation columns is needed. For this purpose, an optimization-based empirical methodology is 
split quaternary mixtures as presented in [13], nevertheless the procedure is adaptable to any other kind 54 of distillation columns.

55 This contribution is organized as follows. First, a brief introduction into the theoretical background is given in section 2. Afterwards, an example case is introduced in order to emphasize the limitations of the original

$57 \dot{V}_{\min }$ diagram as estimation tool for lower total stage numbers in section 3 . With the help of this example, 58 the procedure for the new extended approach is presented in section 4. For this purpose, the so-called 59 stage-adapted $\dot{V}_{\min }$ diagram is introduced. The diagram can be calculated by a heuristically developed 60 approximation approach, thus no flowsheet simulator is required. Last, the original and stage-adapted $61 \dot{V}_{\min }$ diagrams are used to initialize a simulation of the example case and the resulting product purities are 62 compared in section 5.

\section{Theoretical Background}

64 This article focuses on the separation of four-component mixtures, thus the theoretical foundations for 65 this kind of separation task is summarized here. Quaternary mixture, denoted as A, B, C and D 66 corresponding to the boiling points of the components where $A$ is the light boiler, are conventionally split 67 in a sequence of three distillation column. Then, each column before the last one obtains one final product stream and one consisting of a mixture of the residual components. The last column is then just fed with an almost binary feed and has two final product flows. In Figure 1a one option for the coupling columns, the direct-direct split sequence [14], is shown. In the flow chart, boxes with an $\mathrm{x}$ in the middle indicate column sections equipped with separating internals. The expression column region denotes the sections above and below a feed stream being responsible for a certain product split. However, due to the fact that in column sequences not just the heavy boiling component but also the residuals have to be evaporated, this is not the most energy efficient option for the product separation. The minimal feasible energy operation of this separation task without heat integration can only be realized in a multiple dividing wall column (mDWC) [8], which is shown in Figure 1b. Three dividing walls are applied inside the column which prevent remixing of the intermediate boiling components. Consequently, at an infinite stage number these columns require around $50 \%$ less energy input compared to conventional column sequences $[8,9]$. This lower energy demand comes at the cost of a higher complexity. For the column design, the number of theoretical stages has to defined in 12 instead of just six sections and three vapor and liquid splits at the dividing walls have to be defined additionally. 


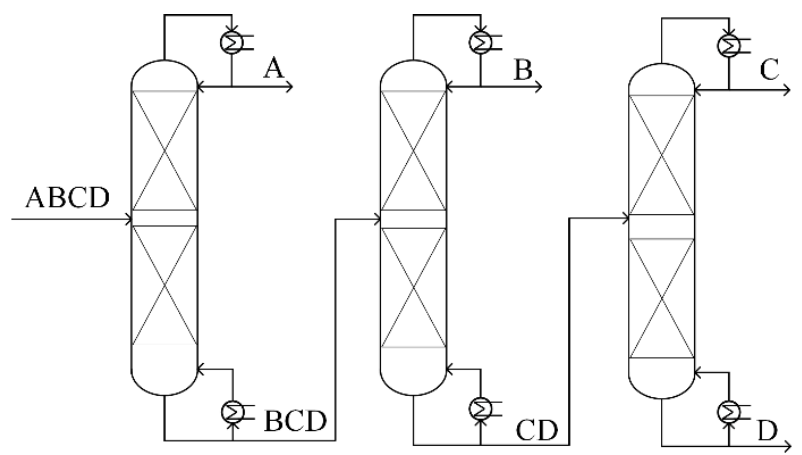

(a)

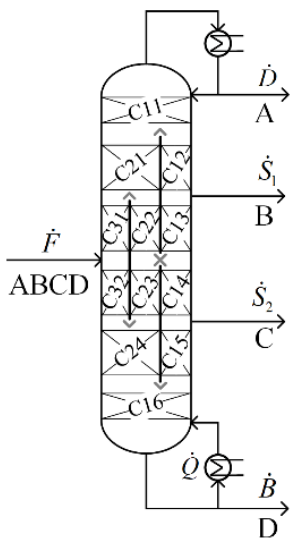

(b)

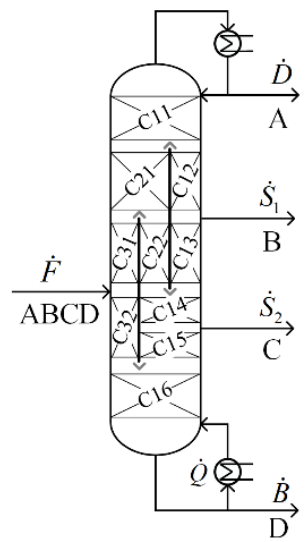

(c)

Figure 1: Options for quaternary product splits. (a) Direct-direct split sequence, (b) multiple dividing wall column and (c) simplified multiple dividing wall column.

However, the complexity can be reduced by neglecting one of the dividing walls, which can be done for many systems without energy penalty [8]. The resulting simplified multiple dividing wall column (smDWC, in literature sometimes 2-2-4 configuration) saves two sections, one liquid and one vapor split.

Similar as conventional distillation columns also the operation of dividing wall columns is limited by the minimum stage operation and the minimum energy operation at an infinite stage number. As a rule of thumb, infinite stages can be approximated at 4 times the minimum one $[15,16]$. Shortcut approaches are available in order to determine these extreme operating points and also optimal compromises in between.

For the minimum stage number estimation the Fenske equation is a well-known approach [17]. The equation is also commonly applied to dividing wall columns [12,18-20].

For the minimum energy demand estimation $\dot{V}_{\min }$ diagrams are a suited tool. They are based on the Underwood equations [21-24] and have been developed by Halvorsen and Skodestad [25-27]. For the calculation of the diagram an infinite stage number, constant relative volatilities and molar flows are assumed. It is a mountain like diagram with maxima and minima. The number of maxima is one below the number of components in the feed stream. An example for a $\dot{V}_{\min }$ diagram of a quaternary system is given in Figure 2a. The diagram shows the minimum vapor demand over the corresponding distillate stream for different product splits in a simple distillation column. For a quaternary feed stream there are three maxima in the diagram, which are denoted as sharp product splits. The first one is called AB split, since all component $A$ from the feed stream is obtained in the distillate stream of the column while all component $B$ is obtained in the bottom stream. The same applies for the following BC and CD separations. In between the maxima there are minima representing sharp product splits with distributing intermediate boiling 
components. An example for this is the AC split, where all component $A$ is in the distillate and all $C$ in the bottom. Meanwhile component B distributes between the product streams. Here the sharp split represents the minimum vapor demand reachable by varying the recovery of component $B$ in the distillate product.
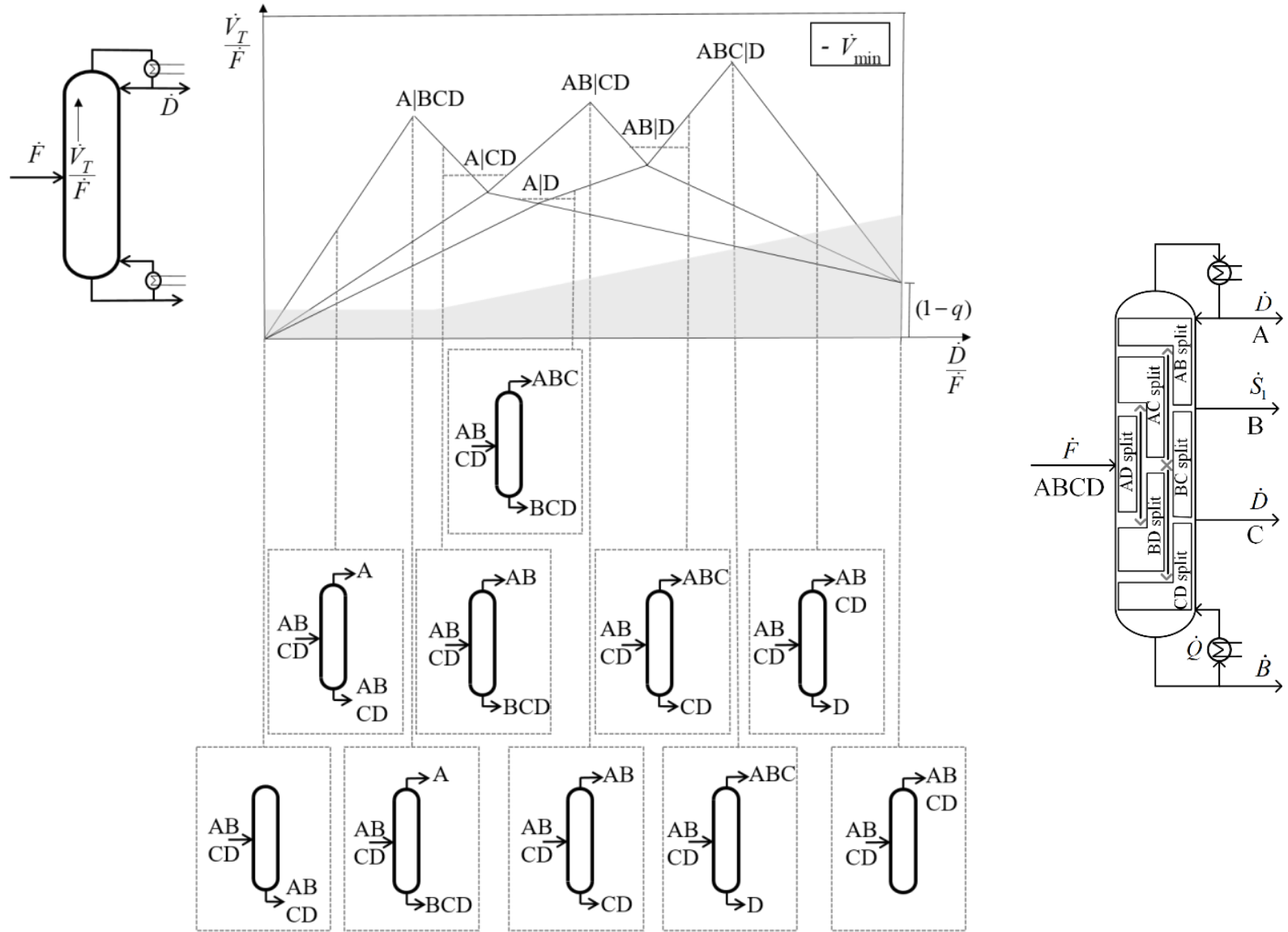

(a)

(b)

Figure 2: (a) Principle of $\dot{V}_{\text {min }}$ diagram for quaternary system and (b) Product split regions in a mDWC.

The $\dot{V}_{\text {min }}$ diagram is directly applicable to a multiple dividing wall column and the highest peak represents the total energy demand of the column. The perquisite for the applicability is that each column region performs its preferred split as shown in Figure 2b. In the section in which the feed stream enters the components $A$ and $D$ are split, the product streams of this separation are fed to regions performing the $A C$ and BD split. The main column part from which the final product flows are obtained performs the product splits without intermediate boiling components.

However, in this contribution a simplified version of the mDWC is considered. For this kind of column the diagram is still applicable with adaptations. Due to the fact that the sections C23 and C24 (see Figure 1c) are missing, the sections C31 and C32 can no more perform their preferred AD split. Instead, the BD split 
119 is performed. As a consequence, in the $\dot{V}_{\text {min }}$ diagram the AB split is shifted, the new shifted peak is often denoted as $A B^{\prime}$, where the superscript prime generally indicates an adjustment of the $\dot{V}_{\text {min }}$ diagram 121 compared to the original one of the feed mixture. As long as the adjusted AB' split stays below the highest 122 peak, the smDWC does not require more energy than the full mDWC [13]. A detailed publication about 123 the calculation of the shifted peaks for such a column can be found elsewhere [10].

124 Due to the fact that the $\dot{V}_{\text {min }}$ diagram does not only show the overall minimum energy demand of the 125 column but also of all column regions performing certain product separations, also the required liquid and 126 vapor splits at the dividing walls can be read [27]. Additionally, it can already be evaluated whether these 127 splits can be operated in a certain range resulting in a more flexible operation. This can be easily derived 128 from a simple mass balance. Obviously, all vapor produced at the bottom of the column will also be 129 available at the top since constant molar flows inside the column are assumed. Correspondingly, all 130 maxima are shifted to the vapor demand of the highest one. As a consequence, also the sharp splits with 131 distributing components are shifted. Nevertheless, the resulting vapor and liquid splits can be operated 132 in between the original and the shifted vapor demand which is then denoted as optimality region [27]. In 133 this work it is distinguished between the optimality region and a flexibility of the liquid and vapor splits. 134 This arises from the fact that the optimality region considers pure products at the minimum energy 135 operation. Flexibility denoted the extended optimality region with a certain distance to the energetic 137 dividing wall columns is quite rare, this topic is explained in more detail in section 3.

138 As already stated, the minimum energy and minimum stage operation are the extreme compromises for the operation of distillation columns. Usually, the design is chosen in between these cases. A well-known 141 which makes use of the standardization of stage number $X$ and reflux ratio $Y$ as shown in equation (1), 142 where $R_{R}$ denotes the reflux ratio. Gilliland states that all optimal column setups are located on one line 143 when plotting $Y$ over $X$. The approach is graphically based.

$$
X=\frac{\sum N_{C i}-\sum N_{\min , C i}}{\sum N_{C i}+1}, Y=\frac{R_{R}-R_{R, \min }}{R_{R}+1}
$$

144 Later, Molokanov extended with approach by a mathematical description of the correlation [29], which is 145 shown in equation (2).

$$
Y=1-\exp \left(\frac{1+54.4 X}{11-117.2 X} \cdot \frac{X-1}{\sqrt{X}}\right)
$$


147 In order to emphasize the limits of the original $\dot{V}_{\min }$ diagram to initialize simulation of smDWCs at finite

148 stage number, an example is first presented here. The $\dot{V}_{\min }$ diagram of a system is calculated and four cases 149 representing the borders of the optimality region are used to initialize a rigorous Petlyuk column 150 simulations provided by Aspen Plus ${ }^{\circ}$. The resulting product purities are then determined.

151 As quaternary mixture ethanol, $n$-propanol, $i$-butanol and $n$-butanol is considered, since it is suggested to 152 be suited for the smDWC in literature [13]. The mixture is assumed to be equimolar and at liquid boiling 153 state, the feed stream is set to $100 \mathrm{~mol} / \mathrm{h}$. For the thermodynamic modelling the non-random two-liquid 154 model (NRTL) is used and suitability of the parameters is validated with experimental data from literature 155 [13]. The relative volatilities of the components are determined to be $\alpha=[4.7,2.2,1.5,1.0]$.

156 The mixture is assumed to be split in a simplified multiple dividing wall column as shown in Figure 1c. This 157 kind of column is to be built at Ulm University with the purpose to be suited for a broad range of mixtures $158[13,30]$. Thus, the theoretical stages in all sections are set equal and for fluid dynamic reasons double in 159 section C32. For the present example, the stage number in all sections is set to 13 as shown in Table 1. In 160 order to quantify that this stage number can be denoted as finite, the minimum stage number for all 161 product splits to reach final product purities of $98 \mathrm{~mol} \%$ are calculated with the Fenske equation. The 162 procedure is presented elsewhere [12]. The factor $n$ is introduced describing the ratio between the actual 163 stage number divided by the corresponding minimum one as shown in equation (3).

$$
n_{i}=\frac{\sum N_{C i}}{\sum N_{\min , C i}}
$$

164 If $n=1$ the minimum stage number is reached while $n=4$ approximates an infinite stage number.

165 Table 1: Stage distribution of the assumed column and minimum stage number for the corresponding product splits for 98 mol\% 166 pure final products

\begin{tabular}{ccccc}
\hline $\begin{array}{c}\text { Sections (see } \\
\text { Figure 1c) }\end{array}$ & Region & $\begin{array}{c}\text { Stage number } \\
\text { in example } \boldsymbol{N}_{\boldsymbol{i}}\end{array}$ & $\begin{array}{c}\text { Minimum stage number } \\
\text { (Fenske) } \boldsymbol{N}_{\text {min, }}\end{array}$ & $\begin{array}{c}\text { Relative stage } \\
\text { number } \boldsymbol{n}_{\boldsymbol{i}}\end{array}$ \\
\hline $\mathrm{C} 11+\mathrm{C} 12$ & $\mathrm{AB}$ & 26 & 12 & 2.2 \\
$\mathrm{C} 13+\mathrm{C} 14$ & $\mathrm{BC}$ & 26 & 24 & 1.1 \\
$\mathrm{C} 15+\mathrm{C} 16$ & $\mathrm{CD}$ & 26 & 22 & 1.2 \\
$\mathrm{C} 21+\mathrm{C} 22$ & $\mathrm{AC}$ & 26 & 17 & 1.5 \\
$\mathrm{C} 31+\mathrm{C} 32$ & $\mathrm{BD}$ & $39(26$ in C32) & 24 & 1.6 \\
\hline
\end{tabular}


168 The factor $n$ for all product splits is below 2.2, thus the stage number is finite. Additionally, it is observable 169 that the factor is different for all product splits, especially the BC split is operated close to the minimum 170 stage number thus $n_{B C}=1.1$. Correspondingly, the stage allocation inside the column sections is also non171 optimal for the system.

172 The original $\dot{V}_{\text {min }}$ diagram of the mixture is shown in Figure 3a. The CD split has the highest peak which 173 defines the total vapor demand of the column. Due to the use of a simplified version of the multiple 174 dividing wall column, the AB peak and correspondingly also the AC minimum is increased as indicated by 175 the dashed line $[10,13,31]$. Note that in the following shifted peaks in comparison to the original $\dot{V}_{\min }$ of 176 the feed stream are indicated by a superscript prime, thus the shift of the AB peak for the smDWC is called $177 A B^{\prime}$. Since the resulting vapor demand of the $A B^{\prime}$ split still requires less energy than the $C D$ split, the 178 simplified column version can be operated without energy penalty. The following Figure $3 b, c$ and $d$ show 179 several options how peaks can be shifted in comparison to the original diagram resulting in the optimality 180 region indicated in Figure 3d.

181 From the optimality regions in Figure $3 a$ to $d$, the borders of the operating ranges for the vapor and liquid 182 splits can be read, the resulting ranges are shown in Figure 7 (section 5). All four cases are then applied to 183 initialize a flowsheet simulation of the considered system in the column from Table 1. 


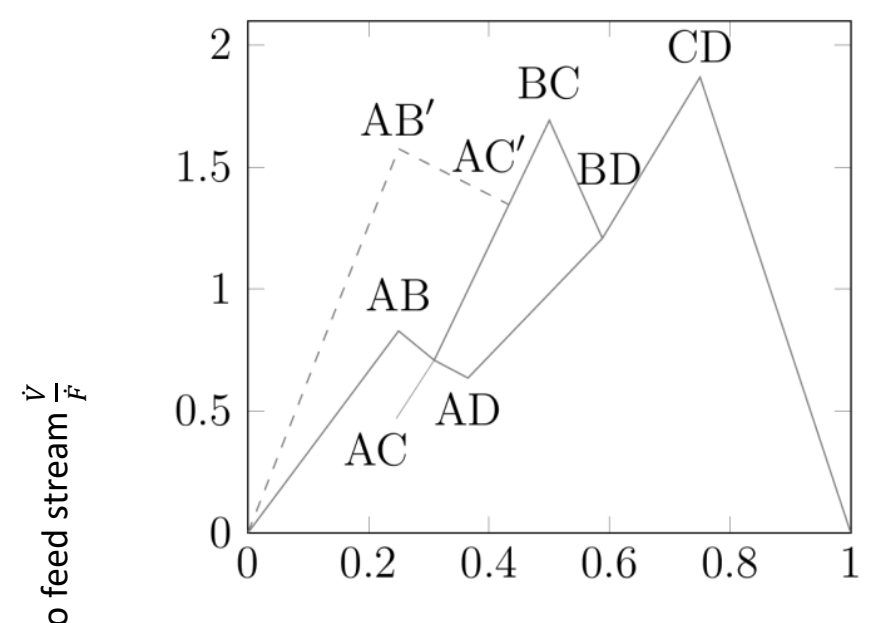

(a)

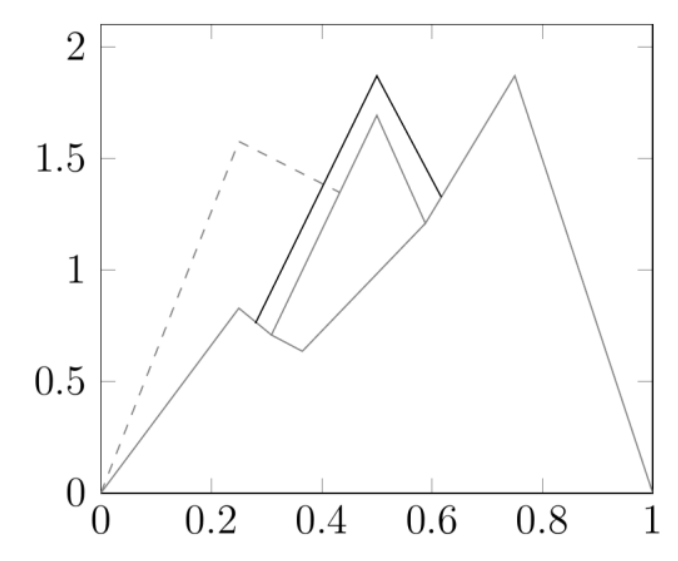

(c)

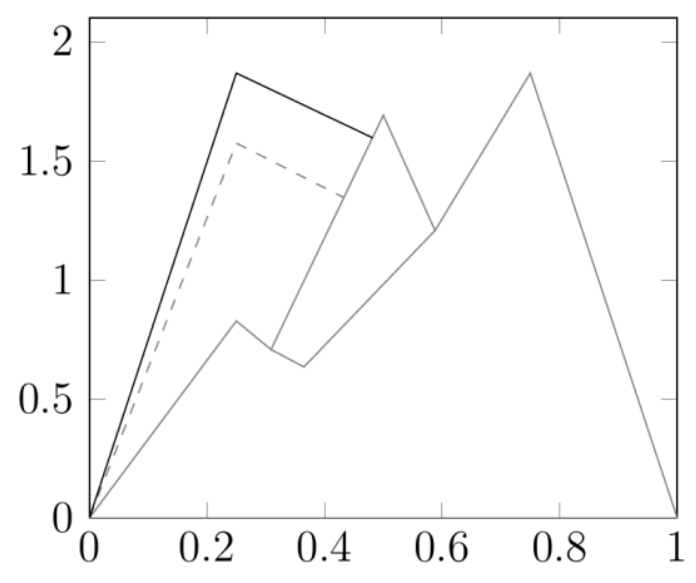

(b)

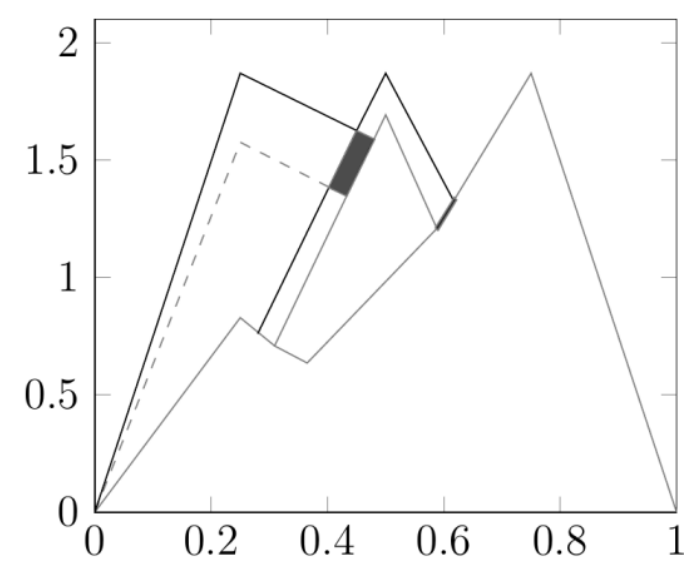

(d)

Figure 3: (a) Original $\dot{V}_{\text {min }}$ diagram for the feed mixture; dashed lines indicate the adaptation of the diagram for the separation in the simplified mDWC version (Figure 1c) and (b)-(d) analytically derived ranges for lower peaks resulting in suitable ranges of the liquid and vapor splits. Gray lines indicate the original $\dot{V}_{\text {min. }}$. diagram, black lines indicate shifted peaks.

Table 2 in section 5 summarizes the resulting product purities for all four cases from Figure $3 a$ to d. In any case, the side draw streams have low product purities around $60 \%$. Correspondingly, it gets clear that the resulting vapor and liquid split ranges are not well suited for the column.

The reason for this observation have already been stated above. On the one hand the overall stage number is relatively low and on the other hand the allocation inside the section is non-optimal. Especially the $B C$ split is operated at a stage number close to the minimum one, while the $A B$ split is operated with more than double the amount of the minimum one. At this point the three fields of application of the $\dot{V}_{\min }$ diagram for estimation of vapor and liquid splits stated in the introduction (section 1) should be recalled. 
The first area where reliable estimates of split ranges are needed is their implementation for real column

operation. Here the guess of the original $\dot{V}_{\min }$ diagram is not sufficient since the objective of columns is usually to obtain product purities above approximately $90 \mathrm{~mol} \%$. The second aspect, to obtain converging flow sheet simulations is partly fulfilled. Even though the simulations converged in the end, in some cases more that the maximal feasible 200 iteration runs set by Aspen Plus had to be used. Last, the third aspect to obtain good initial guesses for an optimization to reach high product purities could not be covered. Actually, an optimization (according to literature ${ }^{29}$ ) initialized with the splits from Figure 3a did not find a global optimum. Here an additional disadvantage of the original $\dot{V}_{\min }$ diagram comes into play: the reboiler input for the finite stage number operation has to be guessed additionally. Overall, it can be concluded that the suitability of the original $\dot{V}_{\min }$ diagram as a universal tool for vapor and liquid split estimations is reduced with decreasing total stage number.

\section{New approach based on extended $\dot{V}_{\min }$ diagram}

207 Nevertheless, to make the basic idea of the $\dot{V}_{\min }$ diagram also usable for lower numbers of theoretical 208 stages a new method is presented here. It is built on the idea of a three-dimensional $\dot{V}_{\min }$ diagram considering also the dimension $n$ (equation(3)), which has been published previously [12]. However, in the original publication an optimization was used to derive the additional dimension of the diagram. In order to avoid the need of an optimization, first a shortcut approach to calculate Pareto-optimal column designs in between the minimum stage number and the minimum energy demand is introduced in section 4.1. Afterwards, the methodology is applied to derive the extended $\dot{V}_{\min }$ diagram of the considered test

214 system in section 4.2. Additionally, inserting the factors $n$ of the column sections into the diagram gives a

215 first hint on the distortion of the $\dot{V}_{\min }$ diagram for the column. In the following section 4.3 the stageadapted $\dot{V}_{\min }$ diagram is introduced. It is a two-dimensional projection of the $\dot{V}_{\min }$ diagram resulting from

217 the column section location in the three-dimensional $\dot{V}_{\min }$ diagram. In this new depiction a graphical 218 approach can be applied to determine adjusted vapor and liquid split ranges for a simulation initialization. 219 In the following section 5 the results derived from the original $\dot{V}_{\min }$ method are compared to the ones 220 based on the new stage-adapted $\dot{V}_{\text {min }}$ method.

\section{$221 \quad 4.1 \quad$ Approximation of Pareto-optimal column designs}

222 To enable a shortcut calculation of the extended three-dimensional $\dot{V}_{\min }$ diagram, a heuristic rule to 223 determine Pareto-optimal distillation column designs between the minimum stage number and minimum 
224 energy operation is required. In order to quantify the suitability of shortcut methods, the suited 225 correlations are compared to a data set derived from rigorous flowsheet optimizations. The data set 226 includes several Pareto-optimal column designs considering the reboiler duty and total stage number of 227 stages as objectives. Optimizations are performed for different binary, ternary or quaternary mixtures 228 that are split in standard distillation columns, column sequences or dividing wall columns. A detailed 229 description of the optimization procedure would exceed the scope of this paper, however the 230 corresponding background has already been published elsewhere [32].

231 As a first step, the Gilliland/Molokanov method which is discussed in section 2 is tested according to its 232 suitability to describe the optimization data. Figure 4a shows the comparison of the scattered 233 optimization results to the shortcut method (black solid line). The optimization results are not well 234 described by the equation. Additionally, the overall scattering range of the data points is relatively large 235 and thus another standardization should be used. Accordingly, a new method is developed here to 236 describe the Pareto-optimal solutions.

237 The procedure to develop the new approximation approach can be distinguished into two steps which are 238 iteratively repeated until a suited result is found. First, a standardization approach has to be found for 239 both dimensions, which are the stage number and energy demand. In this context the reboiler duty is 240 used, however it is similar for the vapor demand or reflux ratio. The aim of normalization is to ensure that 241 all the points considered are located on a line with the smallest possible scattering radius. Second, the 242 mathematical relationship between the two standardized values should be describable by a simple 243 equation.

244 For the new standardization approach, several options are tested. Initially the parameter $n$ shown in 245 equation (3) is assumed. However, after the first iteration with the correlation equation it was decided to 246 additionally divide the number by 0.97 . The reason for this is given in the later part of this chapter, at 247 which the correlation equation is presented. The resulting final standardization of the stage number is 248 shown in equations (4). The standardized energy input is shown in equation (5), however also equation 249 (6) can be applied for the second dimension.

$$
N_{\text {rel }}=\frac{n}{0.97}=\frac{\sum N_{C i}}{0.97 \sum N_{m i n, C i}}
$$

$$
\dot{Q}_{\text {rel }}=\frac{\dot{Q}}{\dot{Q}_{\text {min }}}
$$




$$
\left(\frac{\dot{V}}{\dot{F}}\right)_{\text {rel }}=\frac{\left(\frac{\dot{V}}{\dot{F}}\right)}{\left(\frac{\dot{V}}{\dot{F}}\right)_{\min }}
$$

251 Figure $4 \mathrm{~b}$ shows the location of the same scattered optimization data as Figure $4 a$ but according to the new standardization approach. The scatter radius of the data points is reduced significantly. Interestingly, also no impact of the considered system can be seen.

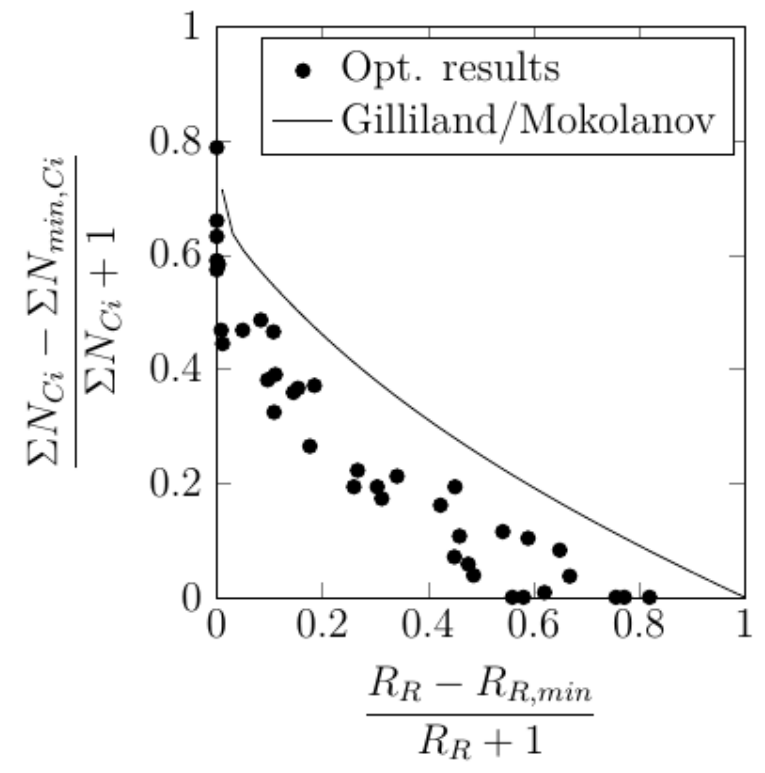

(a)

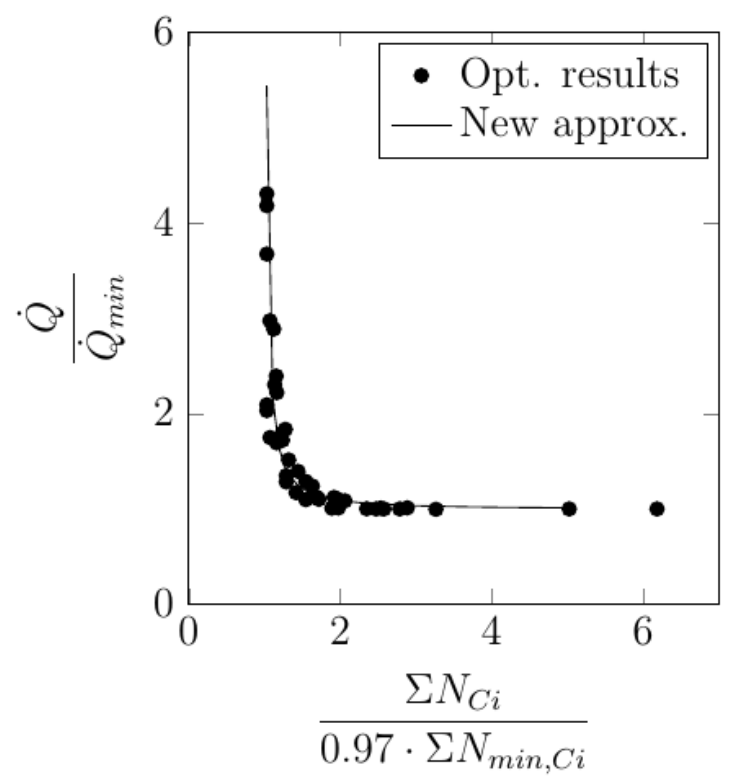

(b)

Figure 4: Standardization methods for Pareto-optimal setups of distillation columns. Comparison of actual optimization results (dots) with heuristic shortcut approaches (lines) (a) Gilliland/Molokanov and (b) new standardization approach and approximation according to equation (8)

A suited correlation to describe the relation between the two dimensions of Pareto-optimal column designs from Figure $4 \mathrm{~b}$ is shown in equation (7). This equation has two asymptotes, $b$ in $\mathrm{x}$-direction and $c$ in y-direction.

$$
\dot{Q}_{r e l}=\frac{a}{N_{r e l}^{2}-b}+c
$$

The parameters $a, b$ and $c$ have to be fitted to the optimization data. At first glance, it is obvious to assume that the minimum energy demand is one asymptote and the other is the minimum stage number. For equation (7) this means that $b$ and $c$ should be one, which results in a quite simple equation. However, the nature of asymptotes is that they are not defined by the corresponding equation. Figuratively speaking, the equation would predict that the minimum stage number and minimum energy demand are not reachable but they actually are. For the heat duty of the evaporator this is no big problem since it is a 

is shown in equation (8).

$$
\dot{Q}_{\text {rel }}=\frac{0.27}{N_{r e l}^{2}-1}+1
$$

274 Correspondingly, by inserting the actual stage number and reboiler duty into the equation the required 275 energy input related to a specific total stage number can be calculated according to equation (9), a similar

276 approach can be used when $\dot{Q}$ is replaced by $\frac{\dot{V}}{\dot{F}}$.

$$
\dot{Q}=\frac{0.27 \dot{Q}_{\min }}{\left(\frac{N}{0.97 N_{\text {min }}}\right)^{2}-1}+\dot{Q}_{\text {min }}
$$

\section{$277 \quad 4.2$ Extended $\dot{V}_{\min }$ diagram by coupling with approximation method}

278 The approximation approach from the previous section 4.1 is applied in order to calculate the three279 dimensional version of the $\dot{V}_{\min }$ diagram without the need of extensive optimization runs. For this purpose, 280 first the original $\dot{V}_{\text {min }}$ diagram is calculated[10]. Then, equation (9) is applied on all considered product 281 splits. For the quaternary system from section 3, the three maxima describing the $A B$ (the increased one 282 for this column version), BC and CD split are required and also the minima for the $A C$ and $B D$ splits. Note 283 that the AD split is not performed in the considered simplified version of the mDWC thus it is not shown 284 here. The resulting diagram for the example system is shown in Figure 5. Additionally, black squares 285 indicate the actual vapor demand for the example system in a column with the assumed non-optimal stage number in the column sections (from Table 1). 


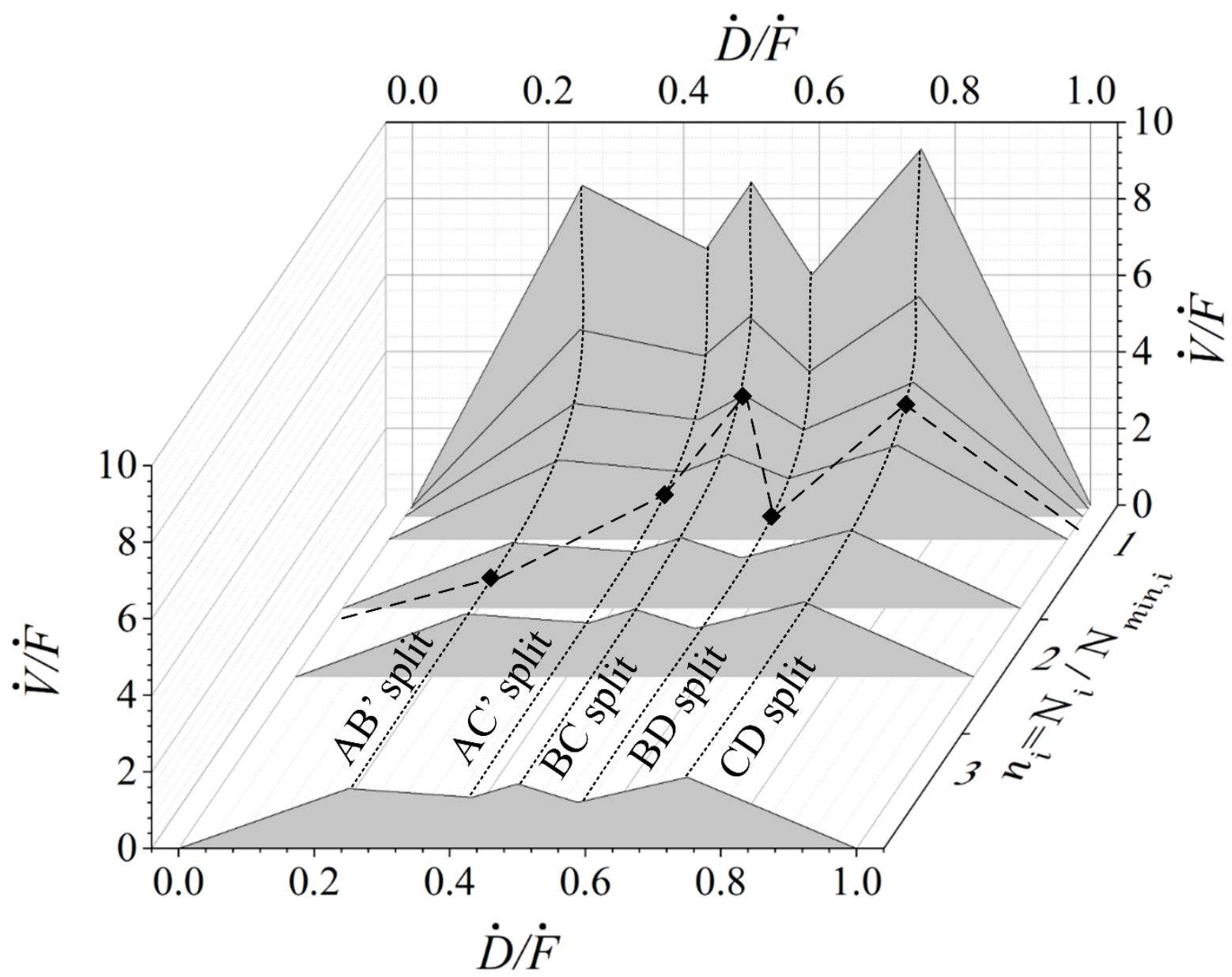

Figure 5: Extended three-dimensional version of the $\dot{V}_{\min }$ diagram for the considered quaternary mixture. Dotted lines: Correlation according to equation (9), black squares and dashed line: determine stage-adapted $\dot{V}_{\text {min }}$ for system inside a column with specified stage allocation.

291 At the front of the diagram at $n_{i}=4$ the original $\dot{V}_{\min }$ diagram can be found. The development of the significant points in the direction to lower $n_{i}$ is calculated with the new approach from section 4.1.

293 Generally, the shape of the $\dot{V}_{\min }$ diagram at lower stage numbers is similar to the one at higher stage 294 numbers. From the markers indicating the example system in the considered column design (Table 1) it 295 already gets clear that the BC split has the highest energy demand of the real column. According to the 296 original $\dot{V}_{\text {min }}$ diagram the CD split is limiting, however this bottleneck is shifted due to the chosen stage 297 allocation in the column sections. Additionally, the AC and BD splits have high relative stage numbers in 298 comparison to the $B C$ split. While the $B C$ split determines the total amount of vapor, the difference 299 between $A C$ and $B D$ to $B C$ sets the flexibility of the vapor and liquid splits. Correspondingly, due to the 300 difference in $n$ of these product splits a relatively broad operating range for the liquid and vapor splits is 301 expectable. 


\subsection{Stage-adapted $\dot{V}_{\min }$ diagram}

303 From the black dashed lines with black markers in Figure 5 a two-dimensional projection to axis similar to

304 the original $\dot{V}_{\min }$ diagram can be obtained which is shown in Figure 6a. The dark gray lines in this diagram

305 are hereafter referred to as stage-adapted $\dot{V}_{\min }$ diagram. All observations from the previous section still

306 hold, however in the two-dimensional projection it is easier to read suited ranges of the vapor and liquid

307 splits. This is indicated in Figure $6 \mathrm{~b}, \mathrm{c}$ and $\mathrm{d}$. As already noted, in contrast to the original $\dot{V}_{\min }$ diagram the

308 stage-adapted $\dot{V}_{\min }$ diagram predicts the BC split to be determining the total energy demand because BC'

309 is the highest peak. Accordingly, to evaluate the flexibility ranges now the $C D$ and $A B$ peaks can be lifted

310 to the height of BC. The determination of the optimality region is performed on a graphical basis.

311 Accordingly, the location of the AC and BD peak have to be guessed. Based on an iterative empirical

312 approach it is found that the location of these minima is best represented when the slopes next to the

313 minima from the original $\dot{V}_{\min }$ diagram are maintained. For an illustration of this description, Figure $6 \mathrm{~b}$ can

314 be considered, in which the CD peak is increased. Correspondingly, the BD minimum has to be estimated.

315 For this purpose, the original $\dot{V}_{\min }$ diagram indicated by the light gray line is considered. The slopes of the

$316 \mathrm{~V}$-shaped line connecting the points BC-BD-CD is graphically shifted to the stage-adapted BC and shifted

$317 \mathrm{CD}$ peaks $\left(\mathrm{BC}^{\prime}-\mathrm{BD}^{\prime \prime}-\mathrm{CD}^{\prime \prime}\right)$. This procedure is repeated for the $A B$ peak in Figure $6 \mathrm{c}$. In between the four

318 cases the ranges of the liquid and vapor splits can be read, which are shown in Figure 7 in the following 319 section 5. 


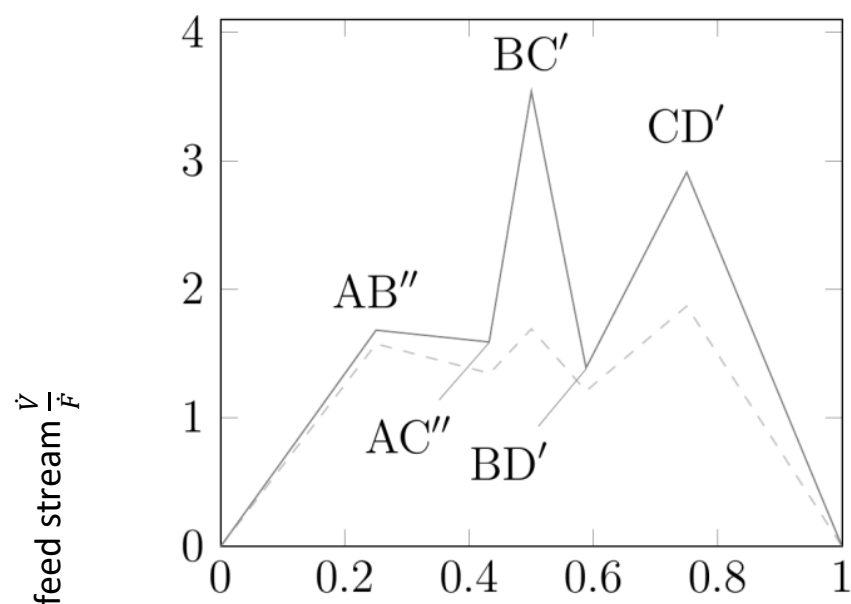

(a)

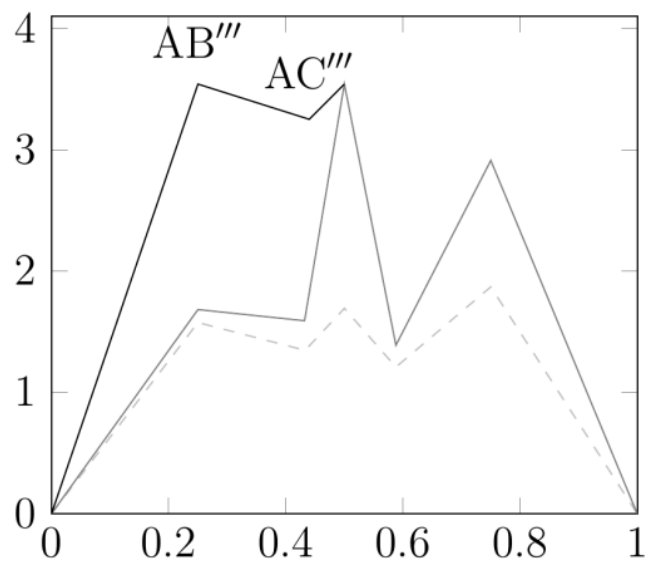

(c)

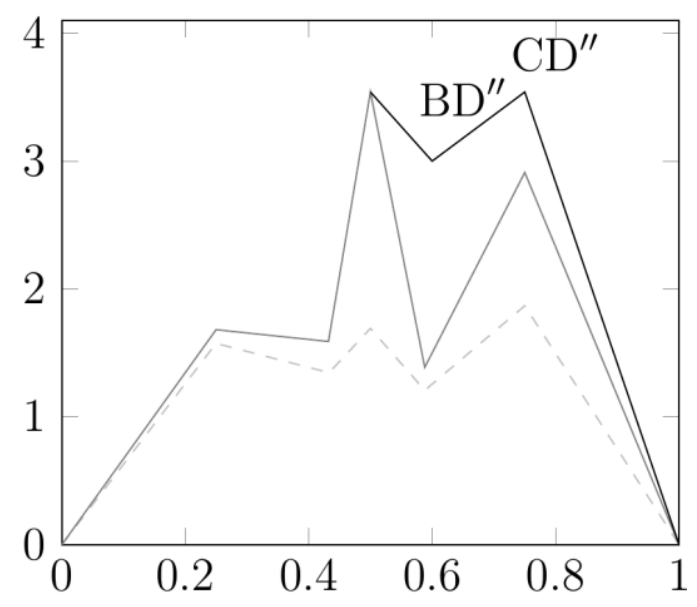

(b)

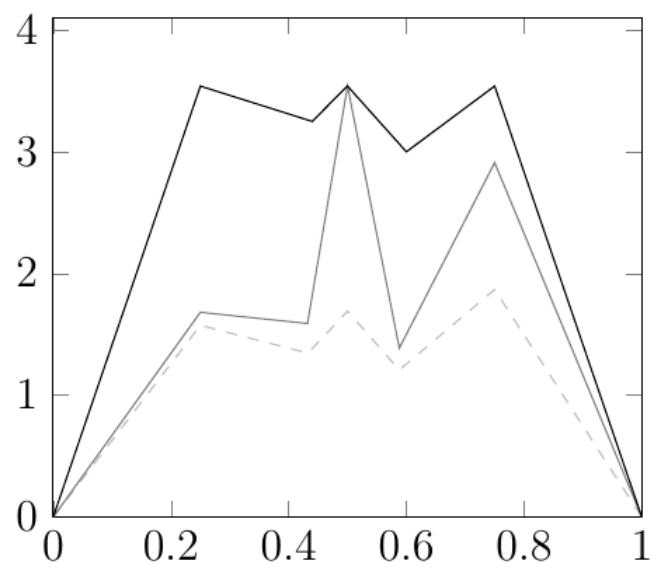

(d)

Distillate stream related to feed stream $\frac{\dot{D}}{\dot{F}}$ $\dot{V}_{\text {min }}$ of mixture in smDWC - Stage-adapted $\dot{V}_{\min } \quad$ Feasible ranges Figure 6: Stage-adapted $\dot{V}_{\text {min }}$ diagram two-dimensional projection of points from Figure 5) and graphical approach to determine vapor and liquid split ranges in (b) to (d).

\section{Comparison of new and old approach}

Figure 7 shows the liquid and vapor split ranges derived by different methods. The shortcut results from the original $\dot{V}_{\min }$ diagram (section 3) and the ones predicted with the stage-adapted version (section 4.3) are compared to results obtained from automated screenings based on rigorous flowsheet simulations (method published elsewhere [12]). Note that the resulting split ranges are four-dimensional, thus two projections of the results are shown here. Also, the section labeling from Figure $1 \mathrm{c}$ has to be kept in mind. The column C2 denoted sections C21 and C22, in which the AC split is performed (dotted lines in Figure 7a). C3 denotes the sections C31 and C33 performing the BD split (solid lines in Figure 7a). Note that an 
adapted split is shown for the liquid split to C3. The adapted split is the product of the two liquid splits to

331 C2 and C3. It is observed that the resulting splits are located on a straight line and thus this representation

332 is chosen. The correlation between columns $\mathrm{C} 2$ and C3 can be seen in Figure 7b. All liquid and vapor splits 333 are defined as the molar flow to the left side of the dividing walls according to Figure $1 \mathrm{c}$ related to the 334 total flow split (index I).

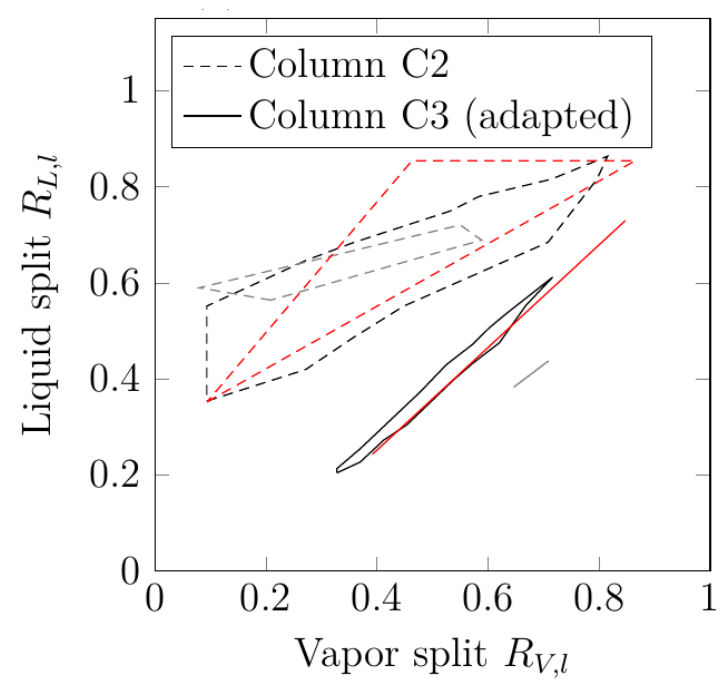

(a)

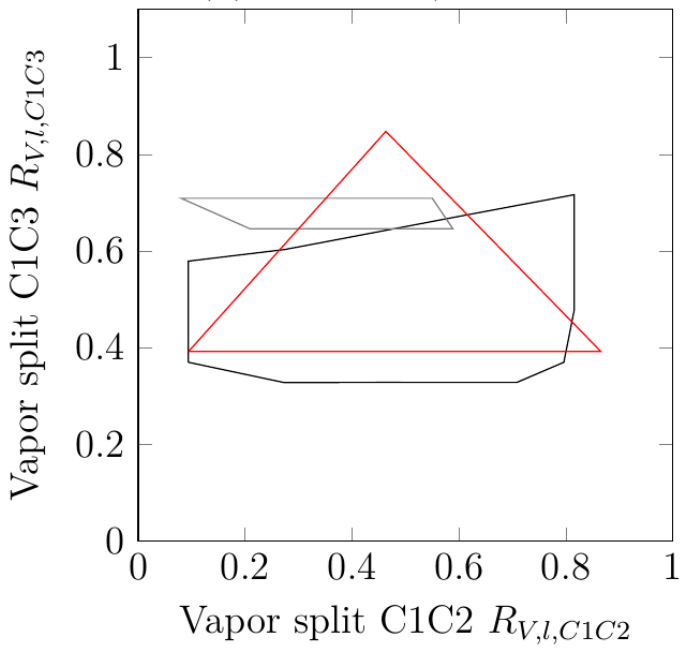

(b)

Figure 7: Actual split ranges for the considered example (black) vs. ranges predicted by original (gray) and adapted (red) $\dot{V}_{\text {min }}$ diagram. Screening results are obtained at $\dot{Q}=1.2 \cdot \dot{Q}_{\text {min }}$ to reach 97 mol\% pure products.

Black lines indicate the actual split ranges based on rigorous flowsheet simulations and gray lines indicate the ranges predicted by the original $\dot{V}_{\min }$ diagram. While the ranges for $\mathrm{C} 2$ fit well with the actual ranges, the liquid split for C3 is significantly underestimated. Note here that the $\dot{V}_{\min }$ diagram predicts a line for the vapor and liquid splits in C3 while a narrow area results from the screening. This behavior can be explained with the fact that lower product purities and an increased energy demand above the minimum one are assumed for the screening. Thus, the corresponding split ranges widen to a field are easier to find compared to a straight line, which is actually valid at the energetic minimum for pure products (from Figure $3 b)$. Also, the correlation between the vapor splits $C 2$ and C3 is not located inside the suited range. This is the reason why the simulations initialized with the original $\dot{V}_{\min }$ shows low purities of the side products (Table 2). In contrast to this, the ranges derived with the new approach (red lines) represent most of the actual range well. Only the upper region of the vapor split to C3 and the upper range of the liquid split to C2 are overestimated. 
349 These extreme cases predicted with the stage-adapted $\dot{V}_{\min }$ diagram are again used to initialize flowsheet 350 simulations of the system introduced in section 3. The resulting product purities are also shown in Table 351 2. It should be noted that the option from Figure $6 \mathrm{~b}$ is not realizable in the column since the BD split 352 cannot be operated at a higher energy input than AC. Consequently, the column section C22 would have 353 to be operated at a negative vapor input. Thus, this option is not considered in Table 2.

354 With the new approach, significantly higher product purities are reached of at least 93.9 mol\% for all 355 products. The case from Figure $6 \mathrm{~d}$ has the lowest purities, the corresponding data point in Figure 7 is the 356 one overestimating the split range for C3. With the cases from Figure $7 \mathrm{a}$ and $\mathrm{b}$ higher purities are reached.

357 Table 2: Comparison of product purities resulting from the two initialization methods

\begin{tabular}{cccccccc}
\hline Component $\boldsymbol{i}$ & \multicolumn{3}{c}{ Original $\dot{\boldsymbol{V}}_{\min }$ method } & \multicolumn{3}{c}{ stage-adapted $\dot{V}_{\text {min }}$ method } \\
\cline { 2 - 8 } & Figure & Figure 3b & Figure & Figure 3d & Figure & Figure & Figure 6d \\
& $3 a$ & & $3 c$ & & $6 a$ & $6 c$ & \\
\hline A & 98.3 & 97.0 & 98.0 & 98.1 & 98.4 & 98.0 & 98.1 \\
B & 66.2 & 66.2 & 61.6 & 63.3 & 96.6 & 97.8 & 94.7 \\
C & 59.9 & 61.5 & 55.9 & 57.7 & 95.7 & 97.1 & 93.9 \\
D & 92.4 & 92.6 & 92.7 & 93 & 97.5 & 97.3 & 97.3 \\
\hline
\end{tabular}

Correspondingly, the new approach is a significant enhancement for the vapor and liquid split description of systems in columns with finite and non-optimal stage numbers. All three aspects stated in the introduction about the significance of this approach are fulfilled again. High product purities are reached, thus the vapor and liquid split ranges are applicable to a real column operation. Also, the initialization of flowsheet simulation was successful. Last, an optimization was performed [32] with the initial simulation according to Figure $6 \mathrm{c}$. With the new approach, in addition to the liquid and vapor split ranges also the required initial energy demand could be estimated with equation (9). This optimization was successful and found a suited optimum which was not possible with the initial simulation by the original $\dot{V}_{\min } \operatorname{diagram}$.

367 Nevertheless, it should still be kept in mind that the assumption of constant relative volatilities is still valid 368 for the stage-adapted $\dot{V}_{\text {min }}$ diagram. Accordingly, for strongly non-ideal systems the method could still suffer reduced applicability. 
371 This paper focuses on the weaknesses of $\dot{V}_{\min }$ diagrams as a tool to estimate liquid and vapor split ranges

372 for dividing wall columns with finite stage numbers and presents a new approach to overcome them. The

373 initial problem results from a sensitivity of the vapor and liquid splits to the total stage number in the

374 column sections. Correspondingly, for columns with relatively low stage numbers and non-optimal

375 allocation, the $\dot{V}_{\min }$ approach does not result in satisfactory product purities. In order to overcome these

376 drawbacks, this paper presents a new heuristic shortcut method to extend the original $\dot{V}_{\min }$ diagram to

377 finite stage numbers. For this purpose, the idea of the $\dot{V}_{\min }$ diagram is coupled with the knowledge about

378 Pareto-optimal column designs in between the minimum energy and stage operation. For the calculation

379 of Pareto-optimal column setups, a new approximation approach is presented. This approach is not

380 limited to dividing wall columns and can also be applied to any other kind of distillation columns. Based

381 on the original $\dot{V}_{\text {min }}$ diagram and the estimation approach for Pareto-optimal column setups, a stage-

382 adapted $\dot{V}_{\min }$ diagram can be calculated. Afterwards, a graphical approach can be used to determine the

383 actual optimality region of the column. Based on this region suited liquid and vapor splits can be derived.

384 It is shown, that the new approach is able to estimate the actually suited ranges very well.

385 Correspondingly, the resulting product purities are significantly higher compared to the results from the 386 original $\dot{V}_{\min }$ method. 
389 We gratefully acknowledge the funding by Deutsche Forschungsgemeinschaft (DFG), project number 390440334941.

$391 \quad 8 \quad$ Symbols used

$392 \quad 8.1 \quad$ Abbreviations

\begin{tabular}{ll}
\hline Abbreviation & Meaning \\
\hline smDWC & Simplified multiple dividing wall column \\
NRTL & Non-random two liquid model \\
mDWC & Multiple dividing wall column \\
\hline
\end{tabular}

393

$394 \quad 8.2 \quad$ Variables

\begin{tabular}{ll}
\hline Variable/Parameter & Meaning \\
\hline$\dot{V}$ & Molar vapor flow \\
$\alpha$ & Relative volatility \\
$\dot{F}$ & Molar feed flow \\
$\dot{D}$ & Molar distillate flow \\
$a-c$ & Parameters to be fitted for approximation approach \\
$N$ & Number of theoretical stages \\
$R$ & Split ratio \\
$n$ & Relative total stage number
\end{tabular}

395

\subsection{Sub- and superscripts}

\begin{tabular}{ll}
\hline Index & Meaning \\
\hline min & Minimum \\
rel & Relative \\
$\mathrm{Ci}$ & Column or column section/region $i$ \\
$\mathrm{R}$ & Reflux \\
$\mathrm{V}$ & Vapor \\
$\mathrm{L}$ & Liquid \\
\hline
\end{tabular}




\begin{tabular}{ll}
\hline I & left \\
& Shifted energy demand in $\dot{V}_{\min }$ diagram compared to original one of the \\
& feed mixture
\end{tabular}

\section{Table and Figure Captions}

\section{References}

423 [1] D. S. Sholl, R. P. Lively, Nature 2016, 532 (7600), 435-437. DOI: 10.1038/532435a. 
424 [2] J. L. Humphrey, Chemical Engineering Progress 1995, 91 (10).

425 [3] J. L. Humphrey, G. E. Keller, Separation process technology, Chemical engineering books, McGraw Hill, New York 1997.

427 [4] A. A. Kiss, C. S. Bildea, Chemical Engineering and Processing: Process Intensification 2011, 50 (3), 281-292. DOI: 10.1016/j.cep.2011.01.011.

[5] Y. Tian, S. E. Demirel, M. F. Hasan, E. N. Pistikopoulos, Chemical Engineering and Processing: Process Intensification 2018, 133, 160-210. DOI: 10.1016/j.cep.2018.07.014.

[6] I. Dejanović, L. Matijašević, Ž. Olujić, Chemical Engineering and Processing: Process Intensification 2010, 49 (6), 559-580. DOI: 10.1016/j.cep.2010.04.001.

[7] M. A. Schultz, D. G. Stewart, J. M. Harris, S. P. Rosenblum, M. S. Shakur, D. E. O'Brien, Chemical Engineering Progress 2002, 98, 64-71.

[8] I. Dejanović, I. J. Halvorsen, S. Skogestad, H. Jansen, Ž. Olujić, Chemical Engineering and Processing: Process Intensification 2014, 84, 71-81. DOI: 10.1016/j.cep.2014.03.009.

[9] I. Dejanović, L. Matijašević, I. J. Halvorsen, S. Skogestad, H. Jansen, B. Kaibel, Ž. Olujić, Chemical Engineering Research and Design 2011, 89 (8), 1155-1167. DOI: 10.1016/j.cherd.2011.01.016.

[10] L.-M. Ränger, U. Preißinger, T. Grützner, ChemEngineering 2018, 2 (2), 25. DOI: 10.3390/chemengineering2020025.

[11] I. Dejanović, L. Matijašević, Ž. Olujić, Chemical and Biochemical Engineering Quarterly 2011, 25 (2), 147-157.

[12] L.-M. Ränger, L. Trescher, M. von Kurnatowski, M. Bortz, T. Grützner, Chemical Engineering and Processing: Process Intensification 2021, 163, 108365. DOI: 10.1016/j.cep.2021.108365.

[13] U. Preißinger, L.-M. Ränger, T. Grützner, ChemEngineering 2019, 3 (2), 34. DOI: 10.3390/chemengineering3020034.

[14] J. K. Kim, P. C. Wankat, Ind. Eng. Chem. Res. 2004, 43 (14), 3838-3846. DOI: 10.1021/ie030640I.

[15] I. J. Halvorsen, S. Skogestad, Journal of Natural Gas Science and Engineering 2011, 3 (4), 571-580. DOI: 10.1016/j.jngse.2011.06.002.

[16] A. A. Kiss, Advanced distillation technologies: Design, control, and applications, Wiley, Chichester, West Sussex, United Kingdom 2013.

454 [19] K. Muralikrishna, V. Madhavan, S. S. Shah, Chemical Engineering Research and Design 2002, 80 455 (2), 155-166. DOI: 10.1205/026387602753501870. 
[20] N. Ramírez-Corona, A. Jiménez-Gutiérrez, A. Castro-Agüero, V. Rico-Ramírez, Chemical Engineering Research and Design 2010, 88 (10), 1405-1418. DOI: 10.1016/j.cherd.2010.02.020.

[21] A. J. V. Underwood, Journal of the Institute of Petroleum 1945, 111-118.

[22] A. J. V. Underwood, Journal of the Institute of Petroleum 1946, 198-613.

[23] A. J. V. Underwood, Journal of the Institute of Petroleum 1946, 614-626.

[24] A. J. V. Underwood, Ind. Eng. Chem. 1949, 41 (12), 2844-2847. DOI: 10.1021/ie50480a044.

[25] I. J. Halvorsen, S. Skogestad, Ind. Eng. Chem. Res. 2003, 42 (3), 596-604. DOI: 10.1021/ie010863g.

[26] I. J. Halvorsen, S. Skogestad, Ind. Eng. Chem. Res. 2003, 42 (3), 605-615. DOI: 10.1021/ie0108649.

[27] I. J. Halvorsen, S. Skogestad, Ind. Eng. Chem. Res. 2003, 42 (3), 616-629. DOI: 10.1021/ie0108651.

[28] E. R. Gilliland, Ind. Eng. Chem. 1940, 32 (9), 1220-1223. DOI: 10.1021/ie50369a035.

[29] Y. K. Molokanov, T. P. Korablina, N. I. Mazurina, G. A. Nikiforov, Chem Technol Fuels Oils 1971, 7 (2), 129-133. DOI: 10.1007/BF00718702.

[30] U. Preißinger, G. Lukač, I. Dejanović, T. Grützner, Chem. Eng. Technol. 2021, 44 (2), 223-237. DOI: 10.1002/ceat.202000557.

[31] L.-M. Ränger, U. Preißinger, T. Grützner, Chemie Ingenieur Technik 2019, 91 (4), 420-428. DOI: 10.1002/cite.201800080.

[32] L.-M. Ränger, M. von Kurnatowski, M. Bortz, T. Grützner, Computers \& Chemical Engineering 2020, 142, 107059. DOI: 10.1016/j.compchemeng.2020.107059. 
Research Article:

Dividing wall columns require reliable initial guesses for vapor and liquid splits for simulation and operation. This contribution presents a reliable approach to fulfil this need even if the column is non-optimally designed and has finite stage numbers. For this purpose, a stage-adapted $V_{\text {min }}$ diagram is introduced, that can be calculated based on shortcut methods.
Shortcut Method for Initialization of Dividing Wall Columns and Estimating Pareto-Optimal NQcurves

M.Sc. Lena-Marie Ränger, Prof. Dr.-Ing. Thomas Grützner*

Chem. Eng. Technol. 20XX, XX (X), xxxx...xxxxx

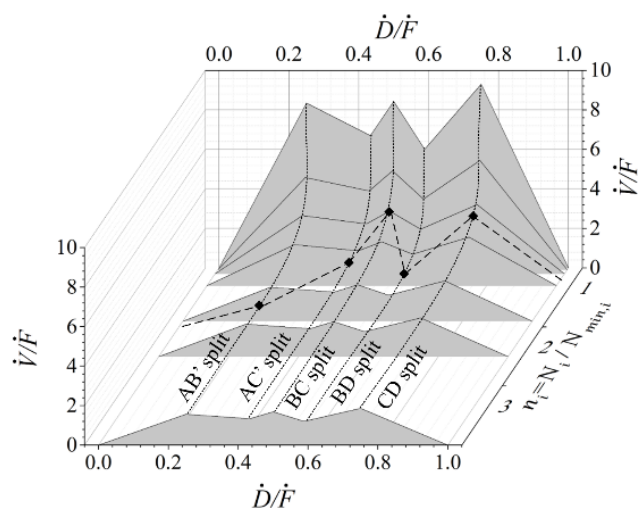

